\title{
Finite-size corrections to the chemical potential
}

\author{
J Ilja Siepmann†ł, Ian R McDonald† and Daan Frenkel§ \\ † Department of Chemistry, University of Cambridge, Lensfield Road, Cambridge \\ CB2 1EW, UK \\ § FOM Institute for Atamic and Molecular Physics, Kruislaan 407, 1098 SJ Amster- \\ dam, The Netherlands
}

Received 25 July 1991, in final form 28 August 1991

\begin{abstract}
The particle-insertion method of Widom has been widely used in numerical simulations for the purpose of calculating the excess chemical potential, $\mu_{\text {ex }}$. It is known, however, that values of $\mu_{e x}$ obtained by Widom's method are strongly dependent on $N$, the number of particles in the system. We use the formalism of the grand-canonical ensemble to derive an expression for the leading term in the finitosize correction to $\mu_{\text {ex }}$. The new expression reduces to the exact result for hard rods and gives numerical results in good agreement with those of simulations of hard-disk and hard-sphere fluids. It is, in both respects, an improvement over an expression previously derived by Smit and Frenkel via a thermodynamic argument.
\end{abstract}

\section{Introduction}

The chemical potential of any species is defined thermodynamically as the change in free energy that occurs when one particle of that species is added to the system of interest. Almost 30 years ago, Widom (1963) showed that the excess chemical potential, $\mu_{\text {ex }}$, of a classical fluid can be calculated from the mean value of the Boltzmann factor associated with the random insertion of a test particle. Widom's result has subsequently provided the basis for most calculations of the chemical potential in Monte Carlo and molecular-dynamics simulations. However, it has been known for some time that the excess chemical potential calculated in this way is strongly systemsize-dependent (Adams 1974, Heinbuch and Fischer 1987). Computer simulations are typically carried out for periodic systems in which the fundamental cell contains of the order of $10^{2}$ to $10^{3}$ particles, and the correction needed to give the infinite-system result can therefore be large. It is always possible to estimate the correction empirically by carrying out simulations for different values of $N$ (the number of particles), but this is very time consuming. It would clearly be much more convenient if the finite-size correction were known explicitly, since it would then be possible to estimate the chemical potential in the thermodynamic limit on the basis of results obtained from simulations of relatively small systems. In a recent paper, Smit and Frenkel (1989) derived an expression for the leading (order $N^{-1}$ ) $N$-dependence of $\mu_{\mathrm{ex}}$. They followed an argument similar to one used by Vrij (1985) in a novel derivation of the

$\ddagger$ Present address: IBM Research Division, Zürich Research Laboratory, Säumerstrasse 4, CH-8803 Rüschlikon, Switzerland. 
Ornstein-Zernike compressibility relation. The basic idea is to interpret the chemical potential as the reversible work needed to add a particle to the system; this work is larger for insertion of a particle into a finite volume than for insertion into an infinite system at the same density. Smit and Frenkel find that to the lowest order in $N^{-1}$ the correction to the chemical potential is

$$
\Delta \mu_{\mathrm{ex}}(N)=\frac{1}{2 N}\left(\frac{\partial P}{\partial \rho}\right)\left[1-k_{\mathrm{B}} T\left(\frac{\partial \rho}{\partial P}\right)\right]^{2}
$$

where $\rho=N / V$ (in three dimensions) is the number density. The appropriate correction can therefore be calculated if the equation of state of the system is known. For a fluid of hard spheres of diameter $d$, the equation of state, $Z(\eta)=\beta P / \rho$, and the compressibility are related by

$$
\beta\left(\frac{\partial P}{\partial \rho}\right)=\frac{\mathrm{d}}{\mathrm{d} \eta}[\eta Z(\eta)] .
$$

where $\beta=\left(k_{\mathrm{B}} T\right)^{-1}$ and $\eta=N \pi d^{3} / 6 V$ is the packing fraction. In their numerical work Smit and Frenkel used the Carnahan-Starling equation of state (Carnahan and Starling 1969) to estimate the finite-size corrections to the excess chemical potential of hard spheres at a number of densities, and compared their results with those obtained by Monte Carlo simulation. Fair agreement was found, but the uncertainties in the Monte Carlo results meant that the comparison was not wholly conclusive.

It is of considerable interest to determine whether equation (1) is generally applicable. As we shall see below, this is not the case. In particular, we show in appendix $A$ that $\Delta \mu_{\text {ex }}(N)$ can be calculated analytically for a one-dimensional system of hard rods of length $d$ in a container of length $L$, often called the Tonks gas (Tonks 1936). The result depends on the boundary conditions; for the periodic Tonks gas we find that

$$
\beta \Delta \mu_{\text {ex }}(N)=\frac{1}{2 N}\left(\frac{\eta}{1-\eta}\right)^{2}
$$

where the packing fraction is now defined as $\eta=N d / L$. On the other hand, the derivation of equation (1) is independent of the dimensionality of the system. In principle, therefore, it should be possible to apply equations (1) and (2) to the hardrod problem, with the density re-defined as $\rho=N / L$. Use of the known, exact, hard-rod equation of state, equation (A1), in (1) and (2) gives

$$
\beta \Delta \mu_{\mathrm{ex}}(N)=\frac{1}{2 N}\left(\frac{1-(1-\eta)^{2}}{1-\eta}\right)^{2}
$$

which is larger than the exact result $(3)$ by a factor $(2-\eta)^{2}$. We have, in addition, carried out a series of Monte Carlo calculations on systems of hard disks of diameter $d$ in an area $A$, for which $\rho=N / A$ and $\eta=\pi d^{2} / 4 A$, and again find significant deviations from the predictions of equation (1).

Prompted by the discrepancies noted above, we have developed a different approach to the derivation of a theoretical expression for the finite-size correction. We show in Appendix $B$ that $\Delta \mu_{\text {ex }}(N)$ can be obtained directly from consideration of the difference in reversible work needed to insert a particle into systems of the canonical 
and grand-canonical ensembles at the same volume and temperature. The leading term is now

$$
\Delta \mu_{\mathrm{ex}}(N)=\frac{1}{2 N}\left(\frac{\partial P}{\partial \rho}\right)\left[1-k_{\mathrm{B}} T\left(\frac{\partial \rho}{\partial P}\right)-\rho k_{\mathrm{B}} T \frac{\left(\partial^{2} P / \partial \rho^{2}\right)}{(\partial P / \partial \rho)^{2}}\right]
$$

This differs from the expression derived by Smit and Frenkel (equation (1) of the present paper) but has the new and encouraging feature of reducing to the exact result for the periodic Tonks gas (equation (3)). In Section 3, we show that the predictions of equation (5) are also in quantitative agreement with the results of Monte Carlo simulations of the two-dimensional hard-disk fluid (this work) and with those of earlier hard-sphere simulations (Smit and Frenkel 1989, Adams 1974). First, however, we consider the results of calculations for the hard-rod system.

\section{The hard-rod fluid}

Although the derivation of equation (3) is exact, the quoted result is correct only to order $N^{-1}$. It is not clear to what extent the neglect of higher-order terms is justified for the system sizes typically used in simulations. As a test of this assumption, we have carried out a series of Monte Carlo calculations for the periodic Tonks gas in which the excess chemical potential of the fluid was calculated by Widom's insertion method. A number of system sizes $(10 \leqslant N \leqslant 400)$ were studied at three different densities, corresponding to packing fractions $\eta=0.4,0.5$ and 0.6 . The initial configuration was in each case taken as one in which the $N$ hard rods were regularly distributed with a spacing equal to $L / N$, and the system was allowed to equilibrate before the calculation of the chemical potential was attempted. The maximum displacement of the rods during a translational Monte Carlo move was chosen so as to yield an acceptance ratio of approximately $50 \%$. In the calculation of $\mu_{\text {ex }}$, each Monte Carlo cycle was followed by $N_{\text {try }}(\approx N / 2)$ insertions of a test particle at random positions, a cycle being defined as $N$ attempts to move randomly chosen particles. Details of the calculations and the results obtained for $\mu_{\mathrm{ex}}$ are given in table 1 . The statistical uncertainties quoted in the table were estimated in the usual way, i.e. by dividing the total simulation into blocks of equal length and calculating the standard error of the mean of the block averages. The $N$-dependence of $\mu_{\mathrm{ex}}$ is illustrated in figure 1 , together with results calculated from equations (3) and (4). As expected the values of $\mu_{\text {ex }}$ obtained by simulation vary approximately linearly with $1 / N$; the magnitude of the $N$-dependent correction increases with increasing density both in absolute terms and relative to the $N \rightarrow \infty$ limit. To allow a more quantitative comparison between theory and simulation, a weighted, linear, least-squares fit (see, for example, Squires (1968)) to a function of the form $a+b / N$ has been carried out on the results of table 1 . The value of the intercept $a$ is the excess chemical potential in the infinite-system limit, $\mu_{\mathrm{ex}}^{\infty}$, for which the exact result is given by equation (A4). The Monte Carlo result for $N=10, \eta=0.6$ was excluded from the fit because it deviates significantly from the best straight line through the other points. It would not be surprising if, at such a small value of $N$, there were an appreciable contribution to $\Delta \mu_{\mathrm{ex}}(N)$ from the term of order $N^{-2}$. The fitted lines are also plotted in figure 1 and the results are summarized in table 2. Agreement with the exact results of equations (A4) and (3) is excellent. Hence, except possibly for very small systems $(N \approx 10$ or less) at high density, the neglect of terms of order $N^{-2}$ in $\Delta \mu_{\text {ex }}(N)$ is justified. 


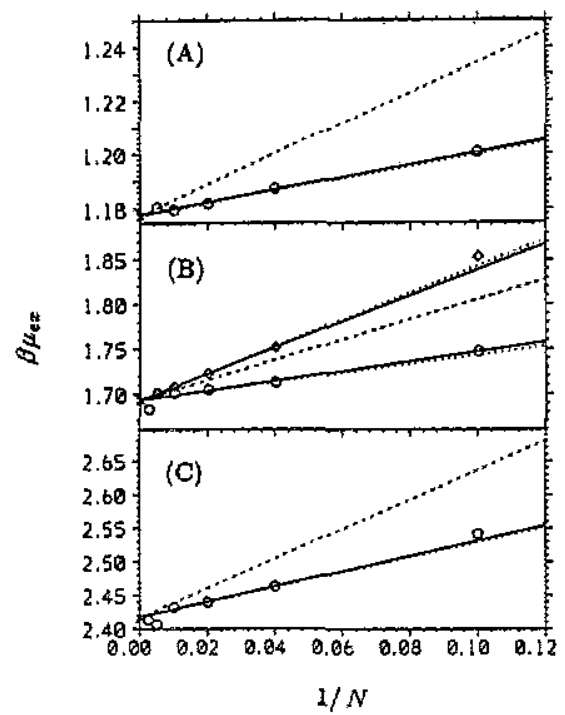

Figure 1. The excess chemical potential $\beta \mu_{\text {ex }}$ of the Tonks gas as a function of the reciprocal number of particles for $\eta=0.4(\mathrm{~A}), 0.5(\mathrm{~B})$, and $0.6(\mathrm{C})$. Opencircles and diamonds show the simulation results for the periodic and hard-wall cases, respectively, and the crosses give the theoretical values calculated from equation (A4). The full lines are obtained from weighted linear fits of the simulation results; the broken lines are calculated from equation (4) and the dotted lines from equations (3) and (A9) for the periodic and hard-wall cases, respectively.

Table 1. Summary of the Monte Carlo simulations of the periodic Tonks gas: $\eta$ is the packing fraction; $N$ is the number of particles; $M C_{e q}$ and $\mathrm{MC}_{\mathrm{pr}}$ are the numbers of equilibration cycles and production cycles, respectively; $N_{\text {try }}$ is the number of attempts to insert a test particle after each cycle; and $\mu_{\text {ex }}$ is the excess chemical potential.

\begin{tabular}{rrrrrl}
\hline$\eta$ & $N$ & $10^{-3} \mathrm{MC}_{\mathrm{eq}}$ & $10^{-3} \mathrm{MC}_{\mathrm{pr}}$ & $N_{\text {try }}$ & $\beta \mu_{\text {ex }}$ \\
\hline 0.4 & 10 & 200 & 500 & 10 & $1.20057 \pm 0.00033$ \\
& 25 & 50 & 750 & 20 & $1.18727 \pm 0.00074$ \\
& 50 & 150 & 500 & 25 & $1.18195 \pm 0.00054$ \\
& 100 & 50 & 250 & 50 & $1.1797 \pm 0.0010$ \\
& 200 & 15 & 100 & 100 & $1.18052 \pm 0.00092$ \\
0.5 & 10 & 110 & 1000 & 10 & $1.74724 \pm 0.00060$ \\
& 25 & 50 & 750 & 20 & $1.71287 \pm 0.00086$ \\
& 50 & 110 & 500 & 25 & $1.70478 \pm 0.00090$ \\
& 100 & 55 & 250 & 50 & $1.7011 \pm 0.0012$ \\
& 200 & 15 & 100 & 100 & $1.7010 \pm 0.0013$ \\
& 400 & 9 & 50 & 200 & $1.6829 \pm 0.0017$ \\
0.6 & 10 & 200 & 500 & 10 & $2.5416 \pm 0.0024$ \\
& 25 & 50 & 750 & 20 & $2.4633 \pm 0.0015$ \\
& 50 & 150 & 500 & 25 & $2.4393 \pm 0.0019$ \\
& 100 & 50 & 250 & 50 & $2.4317 \pm 0.0016$ \\
& 200 & 15 & 150 & 100 & $2.4064 \pm 0.0064$ \\
& 400 & 10 & 45 & 200 & $2.4130 \pm 0.0056$ \\
\hline
\end{tabular}

Also plotted in in figure 1 are the results obtained from non-periodic, hard-wall 
Table 2. Values of the infinite-system excess chemical potential, $\beta \mu_{e x}^{\infty}$, and finitesize corrections, $N \beta \Delta \mu_{\text {ex }}(N)$, for the periodic Tonks gas: $\eta$ is the padking fraction, $M C$ denotes the Monte Carlo results; and $\tau$ denotes results obtained from the exact equations (A4) and (3).

\begin{tabular}{|c|c|c|c|c|}
\hline \multirow[b]{2}{*}{$\eta$} & \multicolumn{2}{|l|}{$\beta \mu_{\text {ex }}^{\infty}$} & \multicolumn{2}{|c|}{$N \beta \Delta \mu_{\text {ex }}(N)$} \\
\hline & $M C$ & $\tau$ & $M C$ & $\tau$ \\
\hline 0.4 & $1.17795 \pm 0.00052$ & 1.177492 & $0.2260 \pm 0.0069$ & 0.2222 \\
\hline 0.5 & $1.6931 \pm 0.0026$ & 1.693147 & $0.539 \pm 0.040$ & 0.5 \\
\hline 0.6 & $2.4177 \pm 0.0037$ & 2.416291 & $1.14 \pm 0.13$ & 1.125 \\
\hline
\end{tabular}

simulations of the Tonks gas at $\eta=0.5$. The best straight line through the data points extrapolates to give essentially the same estimate of $\mu_{\mathrm{ex}}^{\infty}$ as in the periodic case, but the $N$-dependence is now much stronger. The agreement with the corresponding exact result, equation (A9), is again very good.

\section{The hard-disk fluid}

No exact results are available for the equation of state or the chemical potential of the hard-disk fluid. However, an equation of state due to Henderson (1975)

$$
Z(\eta)=\frac{1+\frac{1}{8} \eta^{2}}{(1-\eta)^{2}}
$$

is known to give accurate results at densities up to the fluid-solid transition, $\eta \approx$ 0.68 (Alder and Wainwright 1962). The resulting expression for the excess chemical potential is

$$
\beta \mu_{\mathrm{ex}}=-\frac{7}{8} \ln (1-\eta)+\frac{2 \eta}{1-\eta}+\frac{\frac{9}{8} \eta}{(1-\eta)^{2}}
$$

and the system-size correction obtained by substitution of (2) and (6) into (5) is

$$
\beta \Delta \mu_{\mathrm{ex}}(N)=\frac{1}{2 N} \frac{\mathcal{A}}{\mathcal{B}^{3}}\left[1-\frac{\mathcal{B}^{2}}{\mathcal{A}}\left(\mathcal{B}+\frac{4 \eta+\frac{11}{4} \eta^{2}}{\mathcal{A}}\right)\right]
$$

with $\mathcal{A}=1+\eta+3 / 8 \eta^{2}+1 / 8 \eta^{3}$ and $\mathcal{B}=1-\eta$.

To provide a test of the theoretical predictions, new Monte Carlo calculations have been carried out for the hard-disk fluid over a range of densities and $N=25,49,100$, 196 , and 400 . The same general procedures were followed as in the hard-rod case (section 2). The chemical potentials determined for the 100-disk system by Widom's method are plotted in figure 2, together with results calculated from Henderson's equation (7). Excellent agreement is obtained up to $\eta \approx 0.65$, which is close to the freezing transition. The level of agreement is partly fortuitous, since the infinite-system results (see below) are systematically lower than those predicted by equation (7), but 


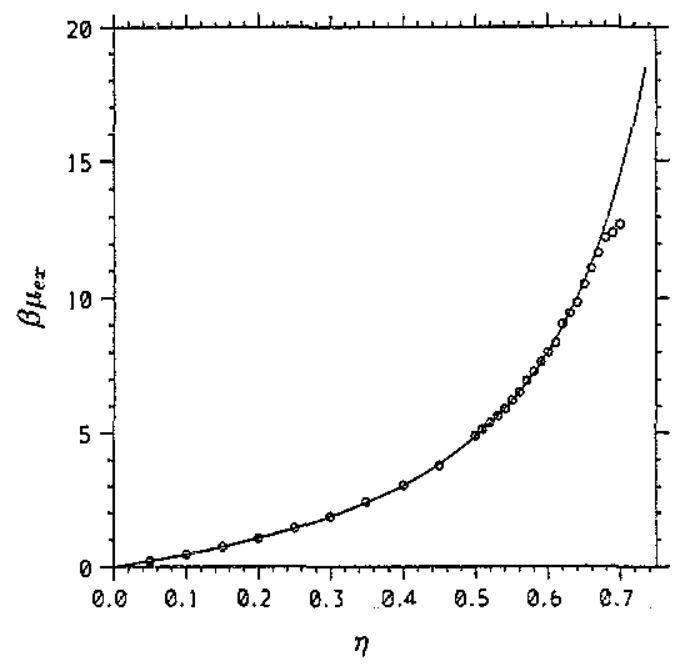

Figure 2. Plot of the excess chemical potential $\beta \mu_{\text {ex }}$ versus the packing fraction $\eta$ for a 100-disk system; the open circles show the simulation results and the solid line is calculated from Henderson's equation (7).

it is clear that use of Henderson's equation of state for the calculation of finite-size corrections from either (1) or (5) should entail only negligible error, at least for $\eta \lesssim 0.6$.

Details of the Monte Carlo simualtions for other values of $N$ are summarized in table 3. Table 4 gives the estimates of $\mu_{\mathrm{ex}}^{\infty}$ and $\Delta \mu_{\text {ex }}(N)$ obtained by fitting the Monte Carlo data in the manner already described for hard rods; the results for $N=25$ and $\eta \geqslant 0.3$ were excluded, since the quality of the linear fit was thereby significantly improved. Table 4 also includes the predicted values of the same quantities, those for $\Delta \mu_{\text {ex }}(N)$ being derived from equation (5). Two sets of results are given, one based on Henderson's equation (6) and the other obtained from an equation of state proposed by Verlet and Levesque (1982), namely

$$
Z(\eta)=\frac{1+\frac{1}{8} \eta^{2}}{(1-\eta)^{2}}-\frac{\frac{1}{32} \eta^{4}}{(1-\eta)^{4}}
$$

Agreement with the Monte Carlo values is very good; only at $\eta=0.6$ is there any substantial disagreement. The two sets of theoretical results differ only slightly from each other; other equations of state (Kratky 1976, 1978, Henderson 1977) that we have tested give results intermediate between these two (Siepmann 1991). The results for $\mu_{\text {ex }}$ at three densities are plotted as functions of $1 / N$ in figure 3 ; it is clear from the figure that equation (5) is far superior to equation (1) in describing the $N$-dependence seen in the simulations.

In view of the good results obtained from equation (5) in both one and two dimensions, we have re-assessed some earlier results for the chemical potential of the hard-sphere fluid. As we have already noted, Smit and Frenkel (1989) found that the $N$-dependence in their results was consistent with the predictions of equation (1), used in conjunction with the Carnahan-Starling equation of state. However, the statistical uncertainties in the Monte Carlo results were large and the system sizes studied were rather small. Moreover, the results obtained for $N=32$ and 64 at the highest density $(\eta=0.366)$ deviate significantly from a linear fit (in $1 / N)$ to earlier data on larger 
Table 3. Summary of the Monte Carlo simulations of the hard-disk fluid: annotations as in table 1.

\begin{tabular}{|c|c|c|c|c|c|}
\hline$\eta$ & $N$ & $10^{-3} \mathrm{MC}_{\mathrm{eq}}$ & $10^{-3} \mathrm{MC}_{\mathrm{pr}}$ & $N_{\text {try }}$ & $\beta \mu_{\mathrm{ex}}$ \\
\hline \multirow[t]{5}{*}{0.05} & 25 & 25 & 600 & 20 & $0.212874 \pm 0.000039$ \\
\hline & 49 & 60 & 500 & 25 & $0.21275 \pm 0.00011$ \\
\hline & 100 & 22.5 & 300 & 50 & $0.21242 \pm 0.00011$ \\
\hline & 196 & 7.5 & 100 & 100 & $0.21233 \pm 0.00015$ \\
\hline & 400 & 36.5 & 62.5 & 200 & $0.21253 \pm 0.00017$ \\
\hline \multirow[t]{5}{*}{0.15} & 25 & 250 & 1000 & 20 & $0.73278 \pm 0.00025$ \\
\hline & 49 & 60 & 1500 & 25 & $0.73087 \pm 0.00019$ \\
\hline & 100 & 20 & 400 & 50 & $0.72999 \pm 0.00019$ \\
\hline & 196 & 15 & 150 & 100 & $0.72982 \pm 0.00021$ \\
\hline & 400 & 4 & 75 & 200 & $0.72882 \pm 0.00028$ \\
\hline \multirow[t]{5}{*}{0.3} & 25 & 25 & 250 & 20 & $1.88374 \pm 0.00093$ \\
\hline & 49 & 60 & 250 & 25 & $1.8717 \pm 0.0013$ \\
\hline & 100 & 27.5 & 250 & 50 & $1.8635 \pm 0.0010$ \\
\hline & 196 & 13.5 & 90 & 100 & $1.8613 \pm 0.0012$ \\
\hline & 400 & 3 & 15 & 200 & $1.8560 \pm 0.0014$ \\
\hline \multirow[t]{5}{*}{0.4} & 25 & 25 & 250 & 20 & $3.0968 \pm 0.0032$ \\
\hline & 49 & 60 & 250 & 25 & $3.0601 \pm 0.0023$ \\
\hline & 100 & 27.5 & 125 & 50 & $3.0402 \pm 0.0018$ \\
\hline & 196 & 14.5 & 70 & 100 & $3.0259 \pm 0.0029$ \\
\hline & 400 & 3 & 30 & 200 & $3.0317 \pm 0.0030$ \\
\hline \multirow[t]{5}{*}{0.5} & 25 & 25 & 250 & 20 & $4.9959 \pm 0.0067$ \\
\hline & 49 & 60 & 250 & 25 & $4.9206 \pm 0.0043$ \\
\hline & 100 & 27.5 & 125 & 50 & $4.8725 \pm 0.0038$ \\
\hline & 196 & 25.5 & 80 & 100 & $4.8530 \pm 0.0047$ \\
\hline & 400 & 3 & 30 & 200 & $4.8477 \pm 0.0060$ \\
\hline \multirow[t]{5}{*}{0.6} & 25 & 25 & 250 & 20 & $8.573 \pm 0.057$ \\
\hline & 49 & 60 & 1000 & 25 & $8.161 \pm 0.019$ \\
\hline & 100 & 27.5 & 500 & 50 & $7.999 \pm 0.022$ \\
\hline & 196 & 23.5 & 160 & 100 & $7.950 \pm 0.023$ \\
\hline & 400 & 3 & 45 & 200 & $7.911 \pm 0.025$ \\
\hline
\end{tabular}

Table 4. Values of the infinite-system excess chemical potential, $\beta \mu_{\mathrm{ex}}^{\infty}$, and finite-size corrections, $N \beta \Delta \mu_{\text {ex }}(N)$, for the hard-disk fluid: $\eta$ is the packing fraction, Mc denotes the Monte Carlo results and $\mathrm{H}$ and VL denote results derived from equation (5) via the equations of state (6) and (9), respectively.

\begin{tabular}{|c|c|c|c|c|c|c|}
\hline \multirow[b]{2}{*}{$\eta$} & \multicolumn{2}{|l|}{$\beta \mu_{e x}^{\infty}$} & \multirow[b]{2}{*}{ VL } & \multicolumn{2}{|c|}{$N \beta \Delta \mu_{\text {ex }}(N)$} & \multirow[b]{2}{*}{$V_{L}$} \\
\hline & $\mathrm{MC}$ & H & & $\mathrm{MC}$ & H & \\
\hline 0.05 & $0.21237 \pm 0.00009$ & 0.21247 & 0.21247 & $0.0127 \pm 0.0050$ & 0.00927 & 0.00927 \\
\hline 0.15 & $0.72904 \pm 0.00020$ & 0.72871 & 0.72867 & $0.093 \pm 0.010$ & 0.10661 & 0.10678 \\
\hline 0.3 & $1.8557 \pm 0.0014$ & 1.85801 & 1.83676 & $0.79 \pm 0.12$ & 0.66215 & 0.66291 \\
\hline 0.4 & $3.0219 \pm 0.0042$ & 3.03031 & 3.02312 & $1.84 \pm 0.33$ & 1.6903 & 1.67979 \\
\hline 0.5 & $4.8322 \pm 0.0033$ & 4.85650 & 4.82087 & $4.26 \pm 0.26$ & 4.1095 & 3.99301 \\
\hline 0.6 & $7.873 \pm 0.010$ & 8.02050 & 7.84406 & $13.96 \pm 0.75$ & 10.363 & 9.4701 \\
\hline
\end{tabular}

systems (Adams 1974). The situation is summarized in table 5, which shows that the effect of including the results for large systems is to bring the Monte Carlo estimates of $\Delta \mu_{\text {ex }}(N)$ into good agreement with those obtained from equation (5).

Smit and Frenkel (1989) also give some results for the Lennard-Jones fluid, but in 


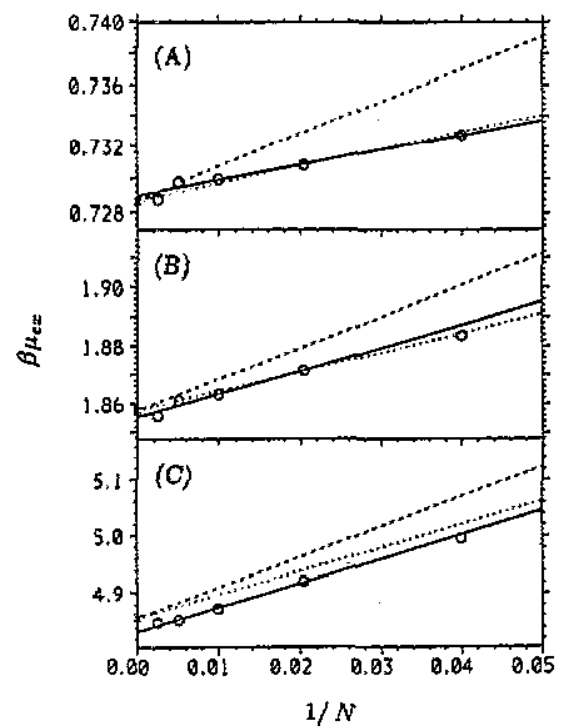

Figure 3. The excess chemical potential $\beta \mu_{\text {ex }}$ of the hard-disk fluid as a function of the reciprocal number of particles for $\eta=0.15(\mathrm{~A}), 0.3(\mathrm{~B})$, and $0.5(\mathrm{C})$; symbols are as in figure 1, with crosses marking values derived from equation (7). The broken lines are calculated from equation (1) and the dotted lines from equation (5), in both cases via Henderson's equation (6).

Table 5. Values of the finite-size corrections, $N \beta \Delta \mu_{\text {ex }}(N)$, for the hard-sphere fluid: $\eta$ is the packing fraction, $\mathrm{BC}_{\mathrm{SF}}$ denotes the Monte Carlo results of Smit and

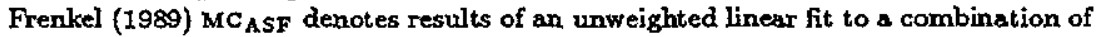
simulation data (Adams 1974, Smit and Frenkel 1989) and $T$ denotes the theoretical predictions derived from equation (5) via the Carnahan-Starling equation of state.

\begin{tabular}{llll}
\hline & \multicolumn{3}{c}{$N \beta \Delta \mu_{\text {ex }}(N)$} \\
\cline { 2 - 4 }$\eta$ & MCSF & $\mathrm{MC}_{\text {ASF }}$ & $T$ \\
\hline 0.262 & $2.3 \pm 0.6^{\mathrm{a}}$ & $2.1^{\mathrm{b}}$ & 2.3 \\
0.314 & $4.7 \pm 0.9^{2}$ & $4.7^{\mathrm{b}}$ & 4.0 \\
0.366 & $9.6 \pm 1.6^{\mathrm{a}}$ & $7.8^{\mathrm{c}}$ & 6.8 \\
\hline
\end{tabular}

$N=32,64$, and 108

b $N=64,108$, and 256

c $N=108,256$, and 864

that case the statistical errors in the simulations are so large as to mask the differences between equations (1) and (5). Comparison between theory and simulation is therefore not illuminating.

\section{Conclusions}

Equation (5) is a new expression for the leading size-dependence of the excess chemical potential, $\mu_{\mathrm{ex}}$, as determined by Widom's method. It differs from an earlier result due to Smit and Frenkel (1989); that result was derived by a thermodynamic argument that now appears to have been incorrect, presumably because fluctuations were not 
properly taken into account. The new expression reduces to the exact result for the one-dimensional, periodic Tonks gas and gives numerical values in very good agreement with those obtained by Monte Carlo calculations for hard disks. The predicted linear dependence of $\mu_{\text {ex }}$ on $1 / N$ does break down when $N$ is sufficiently small, the values at which this occurs being dependent on both density and dimensionality. We find, however, that the linear relation is valid for the system sizes currently used in numerical simulations, i.e. for $N$ of order $10^{2}$ or more. The new expression therefore makes it possible to extrapolate to the thermodynamic limit results for $\mu_{\text {ex }}$ measured for system of, say, 100 particles. Provided a reasonably accurate equation of state is available, the systematic error incurred in this extrapolation appears to be smaller than the statistical errors in the simulations. Equation (5), in combination with the thermodynamic relations given by Smit and Frenkel (1989), also provides a starting point for computation of system-size corrections to pressure and free energy. It could, in particular, be used to correct for finite-size effects on the location of first-order phase transitions, since its derivation is independent of the phase (or dimensionality) of the system of interest.

Attention in this paper has been centred on fluids of hard particles in one and two dimensions. The reasons for concentrating on such simple models are firstly the availability of reliable equations of state, and secondly the fact that that the simulation of systems that are "large" in the relevant sense is computationally more economical in one or two dimensions than in three. Equation (5) itself is of wider applicability. There is, however, a complication in cases where the potential has an attractive part and the derivative $(\partial P / \partial \rho)_{r}$ can therefore vanish. Under these conditions, the expansion leading to equation (B6) cannot be truncated in the manner chosen. Thus equation (5) is not expected to apply near a spinodal or, a fortiori, near a critical point.

\section{Acknowledgments}

We are grateful to the University of Cambridge Computing service for a generous allocation of CPU time, and to the SERC for support under Special Grant No GR/E 68716. One of us (JIS) acknowledges support by a Stipendium der Friedrich-NaumannStiftung aus Mitteln des Ministeriums für Bildung und Wissenschaft der Bundesrepublik Deutschland, and by a Foreign and Commonwealth Office Scholarship. The work of the FOM Institute is part of the research programme of FOM and is supported by the 'Nederlandse Organisatie voor Wetenschappelijk Onderzoek' (NWO).

\section{Appendix A. The Tonks gas}

The equation of state of the Tonks gas is known exactly (Zernike and Prins 1927, Herzfeld and Mayer 1934, Tonks 1936) as

$$
Z(\eta)=\frac{1}{1-\eta}
$$

If $F_{\text {ex }}$ is the excess Helmholtz free energy and $\mu_{\mathrm{ex}}$ is the excess chemical potential, then

$$
\beta F_{\mathrm{ex}}=N \int_{0}^{\eta} \frac{1}{\eta^{\prime}}\left[Z\left(\eta^{\prime}\right)-1\right] d \eta^{\prime}
$$


and

$$
\beta_{\mu_{\mathrm{ex}}}=\beta\left(\frac{\partial F_{\mathrm{ex}}}{\partial N}\right)_{V, T}
$$

Insertion of $(\mathrm{A} 1)$ into $(\mathrm{A} 2)$ and use of $(\mathrm{A} 3)$ gives

$$
\beta \mu_{\mathrm{ex}}=-\ln (1-\eta)+\frac{\eta}{1-\eta}
$$

The system-size dependence of $\mu_{\text {ex }}$ for the Tonks gas can be derived from first principles. Consider first the case of a periodic system. The configurational integral divided by the configurational integral of the ideal gas is

$$
Q_{N} / Q_{N}^{\text {id }}=\left(\frac{L-N d}{L-d}\right)^{N}
$$

The change in excess free energy as the number of particles is increased by one is

$$
\begin{aligned}
\beta \Delta F_{\mathrm{ex}}=-\ln & {\left[\left(\frac{L-(N+1) d}{L-N d}\right)^{N}(1-\eta)\right] } \\
& =-\ln \left[\left(1-\frac{d}{L-N d}\right)^{N}(1-\eta)\right] \\
& =-N\left[-\frac{d}{L-N d}-\frac{1}{2}\left(\frac{d}{L-N d}\right)^{2}+\cdots\right]-\ln (1-\eta)
\end{aligned}
$$

Assuming that $d /(L-N d)$ is small, we find that

$$
\beta \Delta F_{\mathrm{ex}} \approx \frac{\eta}{1-\eta}-\ln (1-\eta)+\frac{1}{2 N}\left(\frac{\eta}{1-\eta}\right)^{2}=\mu_{\mathrm{ex}}^{\infty}+\frac{1}{2 N}\left(\frac{\eta}{1-\eta}\right)^{2}
$$

where $\mu_{\mathrm{ex}}^{\infty}$ is the excess chemical potential in the thermodynamic limit, given explicitly by equation (A4), and the correction term, to order $N^{-1}$, is that given by equation (3) of the main text.

The analogue of (A5) for a system of hard rods confined between hard walls is

$$
Q_{N} / Q_{N}^{\text {id }}=(1-\eta)^{N}
$$

Similar manipulation to that outlined above now gives

$$
\beta \Delta \mu_{e x}(N)=\frac{1}{2 N} \frac{1-(1-\eta)^{2}}{(1-\eta)^{2}} .
$$




\section{Appendix B. A general expression for $\Delta \mu_{\mathrm{ex}}(N)$}

Consider the reversible work required to insert a tagged particle into a volume $V$ in contact with a reservoir of untagged particles at a chemical potential $\mu$. The work required is equal to the change in grand potential and is therefore given by

$$
\begin{aligned}
\beta w_{\mathrm{rev}}=-\ln [ & \sum_{N=0}^{\infty} \frac{\exp (\beta \mu N) f(T)^{N}}{N !} V^{-1} \int \mathrm{d} \boldsymbol{q}^{N+1} \exp \left[-\beta U\left(\boldsymbol{q}^{N}\right)\right] \\
& \times \exp \left[-\beta \Delta U\left(\mathbf{q}_{N+1} ; q^{N}\right)\right] \\
& \left.\times\left(\sum_{N=0}^{\infty} \frac{\exp (\beta \mu N) f(T)^{N}}{N !} \int \mathrm{d} \boldsymbol{q}^{N} \exp \left[-\beta U\left(\boldsymbol{q}^{N}\right)\right]\right)^{-1}\right]
\end{aligned}
$$

where $f(T)$ is the contribution to the canonical partition function that arises from integration over the momenta, $\boldsymbol{q}_{N+1}$ denotes the coordinates of the tagged particle, $\boldsymbol{q}^{N}$ denotes the global coordinates of the remaining particles in the system, and $\Delta U$ is the energy of interaction between the tagged particle and the others. In what follows, we shall replace $f(T) \exp (\beta \mu)$ by the symbol $z$.

The integral in the numerator of equation (B1) is the configurational integral of a system with $N+1$ particles. Thus

$$
\begin{aligned}
\beta w_{\text {rev }}=-\ln \left((z V)^{-1} \frac{\sum_{N=0}^{\infty} \frac{(N+1) z^{N+1}}{(N+1) !} \int \mathrm{d} q^{N+1} \exp \left[-\beta U\left(q^{N+1}\right)\right]}{\sum_{N=0}^{\infty} \frac{z^{N}}{N !} \int \mathrm{d} \boldsymbol{q}^{N} \exp \left[-\beta U\left(\boldsymbol{q}^{N}\right)\right]}\right) \\
=-\ln \left(\frac{\langle N\rangle}{z V}\right)
\end{aligned}
$$

where the second equality is obtained by replacing the dummy index $N$ in the numerator by $N^{\prime} \equiv N+1$. As the chemical potential of an ideal gas at a density $\rho=\langle N\rangle / V$ is $\mu_{\mathrm{id}}(\rho)=k_{\mathrm{B}} T \ln [\rho / f(T)]$, it follows that (B2) can be rewritten as

$$
w_{\text {rev }}=\mu-\mu_{\text {id }}(\langle N\rangle / V) \equiv \mu_{\text {ex }}(\langle N\rangle / V) .
$$

Hence the reversible work required to insert a tagged particle in an open system of volume $V$ is equal to the excess chemical potential of a system at the average density $\rho=\langle N\rangle / V$. The work required is independent of $V$. Thus $w_{\text {rev }}=\mu_{\text {ex }}^{\infty}(\langle N\rangle / V)$, where the superscript $\infty$ denotes a property of an infinite system. With this identification, equation (B1) can be rewritten as

$$
\begin{aligned}
\beta \mu_{\mathrm{ex}}^{\infty}=-\ln \left(\frac{\sum_{N=0}^{\infty} \frac{z^{N} Q_{N}}{N !}\langle\exp (-\beta \Delta U)\rangle_{N V T}}{\sum_{N=0}^{\infty} \frac{z^{N} Q_{N}}{N !}}\right) \\
=-\ln \left(\sum_{N=0}^{\infty} P(N)\langle\exp (-\beta \Delta U)\rangle_{N V T}\right)
\end{aligned}
$$

where

$$
P(N) \equiv \frac{\frac{z^{N} Q_{N}}{N !}}{\sum_{N=0}^{\infty} \frac{z^{N} Q_{N}}{N !}}
$$


is the probability of finding a system of the grand-canonical ensemble in a state with precisely $N$ particles. In other words, $\mu_{e x}^{\infty}(\langle N\rangle / V)$ is equal to the average over all $N$ of the mean Boltzmann factor associated with the addition of a test particle to a system of $N$ particles. The mean Boitzmann factor is the quantity that yields the excess chemical potential in Widom's insertion method (Widom 1963). We shall therefore denote $\langle\exp [-\beta \Delta U]\rangle_{N V T}$ by $\exp \left[-\beta \mu_{e x}^{\text {Wid }}(N, V, T)\right]$.

We now expand $P(N)$ around $(N)$. This, in fact, is a slightly subtle step, because $\langle N\rangle$ is not the same as the most probable value of $N$. However, the difference is important only for corrections of order $N^{-2}$. Denoting $(N-\langle N\rangle)$ by $\Delta N$, we can write

$$
P(N) \approx C \exp \left[-\frac{\beta}{2}\left(\frac{\partial^{2} F(N, V, T)}{\partial N^{2}}\right)(\Delta N)^{2}\right] .
$$

The next step is to expand $\mu_{\text {ex }}^{\text {Wid }}(N, V, T)$ around the average density:

$$
\beta \mu_{\mathrm{ex}}^{\mathrm{Wid}}(N, V, T)=\beta \mu_{\mathrm{ex}}^{\mathrm{Wid}}(\langle N\rangle, V, T)+\beta\left(\frac{\partial \mu_{\mathrm{ex}}}{\partial N}\right) \Delta N+\frac{\beta}{2}\left(\frac{\partial^{2} \mu_{\mathrm{ex}}}{\partial N^{2}}\right)(\Delta N)^{2}+\cdots
$$

and follow this by expanding $\exp \left[-\beta\left(\mu_{\mathrm{ex}}^{\text {Wid }}(N, V, T)-\mu_{\mathrm{ex}}^{\text {Wid }}(\langle N\rangle, V, T)\right)\right]$ to second arder in $\Delta N$ :

$$
\begin{aligned}
\exp \left[-\beta \mu_{\mathrm{ex}}^{\mathrm{Wid}}(N, V, T)\right]=\exp \left[-\beta \mu_{\mathrm{ex}}^{\mathrm{Wid}}((N), V, T)\right]\left[1-\beta\left(\frac{\partial \mu_{\mathrm{ex}}}{\partial N}\right) \Delta N\right. \\
\left.+\frac{1}{2}\left(\beta \frac{\partial \mu_{\mathrm{ex}}}{\partial N}\right)^{2}(\Delta N)^{2}-\frac{1}{2}\left(\beta \frac{\partial^{2} \mu_{\mathrm{ex}}}{\partial N^{2}}\right)(\Delta N)^{2}+O\left(\Delta N^{3}\right)\right] .
\end{aligned}
$$

We also require the following thermodynamic relations:

$$
\begin{aligned}
& \left(\frac{\partial^{2} F}{\partial N^{2}}\right)=N^{-1}\left(\frac{\partial P}{\partial \rho}\right)+O\left(N^{-2}\right) \\
& \left(\frac{\partial \mu_{\text {ex }}}{\partial N}\right)=N^{-1}\left(\frac{\partial P_{\text {ex }}}{\partial \rho}\right)+O\left(N^{-2}\right) \\
& \left(\frac{\partial^{2} \mu_{e x}}{\partial N^{2}}\right)=N^{-2}\left(\rho \frac{\partial^{2} P_{\text {ex }}}{\partial \rho^{2}}-\frac{\partial P_{\text {ex }}}{\partial \rho}\right)+O\left(N^{-3}\right)
\end{aligned}
$$

where $P_{\text {ex }} \equiv P-k_{\mathrm{B}} T$ is the excess pressure. Substitution of equations (B6) and (B8) to (B11) in (B4) gives the desired result, namely

$$
\begin{aligned}
\Delta \mu_{\mathrm{ex}}^{N} \equiv \beta \mu_{\mathrm{ex}}^{W} & (\langle N\rangle, V, T\rangle-\beta \mu_{\mathrm{ex}}^{\infty}(\langle N\rangle, V, T) \\
= & k_{\mathrm{B}} T \ln \left(1-\frac{1}{N}\left(\frac{\partial P_{\mathrm{ex}}}{\partial \rho k_{\mathrm{B}} T}\right) \Delta N+\frac{1}{2 N^{2}}\left\{\left(\frac{\partial P_{\mathrm{ex}}}{\partial \rho k_{\mathrm{B}} T}\right)^{2}\right.\right. \\
& \left.\left.-\left[\rho k_{\mathrm{B}} T\left(\frac{\partial^{2} P_{\mathrm{ex}}}{\partial\left(\rho k_{\mathrm{B}} T\right)^{2}}\right)-\left(\frac{\partial P_{\mathrm{ex}}}{\partial \rho k_{\mathrm{B}} T}\right)\right]\right\}(\Delta N)^{2}+\cdots\right) \\
\approx & k_{\mathrm{B}} T \ln \left\{1+\frac{1}{2 N}\left(\frac{\partial \rho k_{\mathrm{B}} T}{\partial P}\right)\left[\left(\frac{\partial P_{\mathrm{ex}}}{\partial \rho k_{\mathrm{B}} T}\right)^{2}\right.\right. \\
& \left.\left.-\rho k_{\mathrm{B}} T\left(\frac{\partial^{2} P_{\mathrm{ex}}}{\partial\left(\rho k_{\mathrm{B}} T\right)^{2}}\right)+\left(\frac{\partial P_{\mathrm{ex}}}{\partial\left(\rho k_{\mathrm{B}} T\right)}\right)\right]\right\} \\
= & \frac{1}{2 N}\left(\frac{\partial P}{\partial \rho}\right)\left[1-k_{\mathrm{B}} T\left(\frac{\partial \rho}{\partial P}\right)-\rho k_{\mathrm{B}} T \frac{\left(\partial^{2} P / \partial \rho^{2}\right)}{(\partial P / \partial \rho)^{2}}\right]+O\left(N^{-2}\right)
\end{aligned}
$$




\section{References}

Adams D J 1974 Mol. Phys. 281252

Alder B J and Wainwright T E 1962 Phys. Rev. 127359

Carnahan N F and Starling K E 1969 J. Chem. Phys. 51635

Heinbuch U and Fischer J1975 Mol. Simul. 1109

Henderson D 1977 Mol. Phys. 30971 1987 Mol. Phys. 34301

Herzfeld K F and Mayer M G 1934 J. Chem. Phys. 238

Kratky K W 1976 Physica A 85607

1978 J. Chem. Phys. 692251

Siepmann J I 1991 PhD Thesis University of Cambridge

Smit B, and Frenkel D 1989 J. Phys.: Condens. Matter 18659 and references therein

Squires G L 1968 Practical Physica (New York: McGraw-Hill)

Tonks L 1936 Phys. Rev. 50955

Verlet L and Levesque D 1982 Mol. Phys. 46969

Vrij A 1985 Proc. Acad. Sci. Amsterdam B 88221

Widom B 1963 J. Chem. Phys. 392808

Zernike $H$ and Prins J A 1927 Z. Phys. 41184 